\title{
Cell fate conversion by mRNA
}

\author{
Mo Lil ${ }^{1 \dagger}$ Ignacio Sancho-Martinez ${ }^{1+}$ and Juan Carlos Izpisua Belmonte ${ }^{* 1,2}$
}

\begin{abstract}
Recent development of a synthetic mRNA-based technology for efficient reprogramming to

pluripotency and cell fate conversion without any modification to the genome has generated great interest among researchers and clinicians alike. It is hoped that this technology could contribute to unmet needs on several fronts of regenerative medicine, including mechanistic study of reprogramming, generation of safe induced pluripotent stem cells suitable for clinical applications, and derivation of desired cell types for cell-replacement therapy. We will discuss the technological advancements made by this synthetic mRNA methodology, its implications, as well as the challenges that lie ahead in the field of regenerative medicine.
\end{abstract}

It was more than five decades ago when cellular reprogramming was first shown by somatic cell nuclear transfer. These seminal experiments show that somatic cells can revert to pluripotency by somatic cell nuclear transfer, proving the totipotency of their genome. Through later studies we learned that somatic cell fate is mainly driven by a specific set of transcription factors and solidified by epigenetic mechanisms, which can be reverted by reprogramming activities in oocytes or embryonic stem cells. The knowledge gained in the past half a century culminated in the breakthrough discovery of induced pluripotency by Yamanaka and Takahashi in 2006 [1]. They demonstrated that terminally differentiated cells can return to an embryonic-like pluripotent state (termed induced pluripotent stem cells (iPSCs)) by forced expression of four transcription factors (Oct4, Sox2, Klf4 and c-Myc) [1]. iPSC technology has since

\footnotetext{
†These authors contributed equally to this work

*Correspondence: belmonte@salk.edu, izpisua@cmrb.eu

'Salk Institute for Biological Studies, 10010 North Torrey Pines Road, La Jolla, CA 92037, USA

${ }^{2}$ Center of Regenerative Medicine in Barcelona, Doctor Aiguader, 88, 08003, Barcelona, Spain
}

spurred a plethora of studies aimed at understanding the mechanism of reprogramming, modeling human diseases and developing cell-based therapies for degenerative conditions.

Despite great enthusiasm and effort, iPSC-related research is hampered by the fact that iPSC generation is a slow and inefficient process, and that most iPSC derivation protocols entail modifications of the host genome. The most widely adopted method for generating iPSCs relies on integrating retroviral vectors. The process takes approximately 4 weeks and only 0.01 to $0.1 \%$ of the cells become iPSCs. In addition, there are serious concerns regarding the safety of these virally derived iPSCs. The integrated proviruses may cause insertional mutagenesis, bias the differentiation potential of iPSCs if not silenced, and lead to tumor formation once reactivated during the differentiation process [2]. People have tried to avoid these issues by generating transgene-free iPSCs using different strategies, including non-integrative vectors, excisable vectors, and cell-penetrating proteins. The DNA-based methods that are ostensibly nonintegrating still require careful characterization of the iPSC genome to rule out random integration of small fragments of the vector. The removal of excisable vectors entails complex manipulations and prolonged culture, and still leaves a 'scar' in the genome in many cases. Moreover, none of the above mentioned technologies resolve the issue of slow kinetics and low efficiency of iPSC generation [3].

Can the 'three wishes' - safety, speed and efficiency - of reprogramming ever be fulfilled? A recent paper by Warren and colleagues [4] may have just provided an answer. The innovation of the study is centered on the use of synthetic mRNA, a previously underexplored route for delivery of reprogramming factors, in iPSC generation. In contrast to DNA-based vectors, the use of mRNA completely eliminates the risk of modifying the host genome. It has several advantages over the protein transduction approach: it is simpler and more efficient, as one mRNA molecule is likely to undergo multiple rounds of translation before its degradation; and the proteins produced inside the cell have proper post-translational modifications, resulting in more precise localization and higher activity. Indeed, mRNA-based gene delivery has been shown to be highly efficient in human stem and 
progenitor cells [5]. Furthermore, its safety has been demonstrated in clinical trials [6]. However, there are two major roadblocks to adapting this methodology to cellular reprogramming. First, mRNAs are rapidly turned over in the cell, which is incompatible with the requirement for sustained expression of reprogramming factors for about 2 weeks. This point is clearly illustrated in a recent study by Plews and colleagues [7] in which a single electroporation of mRNAs encoding OCT4, SOX2, KLF4, c-Myc and SV40 large T antigen resulted in only partially reprogrammed cells. After careful examination of the kinetics of reprogramming factor levels after mRNA transfection, Warren and colleagues [4] concluded that daily transfection is necessary to maintain the reprogramming activities. Consequently, this strategy leads to the second caveat, which is the high cytotoxicity triggered by repeated transfection of foreign mRNA via an NF-kB-dependent anti-viral pathway. Through careful experimentation, the authors overcame these issues by introducing a series of changes to the standard protocol to reduce the immunogenecity of synthetic RNA (removal of 5' triphosphates, incorporation of modified ribonucleosides) and to suppress interferon signaling pathways (media supplementation of interferon inhibitor B18R). Armed with these innovations, they successfully developed an mRNA-based reprogramming protocol that is two times faster and 35-fold more efficient than the viral one. Moreover, the global gene expression profile of RNA-induced pluripotent stem cells (RiPSCs) more closely resembles human embryonic stem cells than virally derived iPSCs. As the authors pointed out, such a difference may be attributed to the absence of transgenes in RiPSCs. However, it is worth pointing out that other factors, such as the culture conditions that the cells are exposed to during reprogramming as well as the passage number of iPSCs, are known to affect the epigenetic state of iPSCs.

As shown in this study and others, the mRNA-based methodology also applies to directed differentiation of iPSCs and direct fate conversion between terminally differentiated cells. Other than improving efficiency and safety of existing protocols, the mRNA-based technology can aid in the development of better protocols that ensure stable and complete conversion to the desired cell types, which are independent of exogenous factors. In other situations, synthetic mRNAs may be used to alter cell fate temporarily. For example, gene targeting by homologous recombination is extremely inefficient in human iPSCs, but it can be greatly improved in the naïve pluripotent state. However, such a state in human iPSCs requires overexpression of Oct4, Klf4 and Klf2 [8]. It is conceivable that these factors can be delivered as mRNA to induce the naïve pluripotent state without any modifications to the genome.
The synthetic mRNA technology developed by Warren and colleagues represents one of the few examples of major technical advancements in regenerative medicine. It is likely to accelerate the study of the mechanism of cellular reprogramming and the translation of research findings into clinical practice. While being optimistic, we should also remain vigilant in studying the safety of the cells derived by this technology. For example, further studies are necessary to make sure that repeated transfections and prolonged inhibition of interferon signaling do not cause any lasting change in the cells or constitute a selection pressure for cells that are deficient in innate immune response. More importantly, we and others recently showed that iPSCs derived by existing protocols (including RiPSCs) are equally prone to the accumulation and positive selection of mutations in tumor suppressor genes, oncogenes and genes important for cell cycle regulation $[9,10]$. It is then logical to assume that not only does a great deal of research effort have to be focused on the development of efficient nonintegrative approaches but also on novel methods limiting the accumulation of harmful mutations during the reprogramming process.

\section{Abbreviations}

iPSC, induced pluripotent stem cell; RiPSC, RNA-induced pluripotent stem cells.

\section{Competing interests}

The authors declare that they have no competing interests.

\section{Acknowledgements}

Work in the laboratory of JCIB was supported by the IPSEN Foundation.

\section{Published: 9 February 2011}

\section{References}

1. Takahashi K, Yamanaka S: Induction of pluripotent stem cells from mouse embryonic and adult fibroblast cultures by defined factors. Cell 2006, 126:663-676.

2. Miura K, Okada Y, Aoi T, Okada A, Takahashi K, Okita K, Nakagawa M, Koyanagi M, Tanabe K, Ohnuki M, Ogawa D, Ikeda E, Okano H, Yamanaka S: Variation in the safety of induced pluripotent stem cell lines. Nat Biotechnol 2009, 27:743-745.

3. Stadtfeld M, Hochedlinger K: Induced pluripotency: history, mechanisms, and applications. Genes Dev 2010, 24:2239-2263.

4. Warren L, Manos PD, Ahfeldt T, Loh YH, Li H, Lau F, Ebina W, Mandal PK, Smith ZD, Meissner A, Daley GQ, Brack AS, Collins JJ, Cowan C, Schlaeger TM, Rossi $D J$ : Highly efficient reprogramming to pluripotency and directed differentiation of human cells with synthetic modified mRNA. Cell Stem Cell 2010, 7:618-630.

5. Wiehe JM, Ponsaerts P, Rojewski MT, Homann JM, Greiner J, Kronawitter D, Schrezenmeier $\mathrm{H}$, Hombach V, Wiesneth M, Zimmermann O, Torzewski J: mRNA-mediated gene delivery into human progenitor cells promotes highly efficient protein expression. J Cell Mol Med 2007, 11:521-530.

6. Van Nuffel AM, Corthals J, Neyns B, Heirman C, Thielemans K, Bonehill A: Immunotherapy of cancer with dendritic cells loaded with tumor antigens and activated through mRNA electroporation. Methods Mol Biol 2010, 629:405-452.

7. Plews JR, Li J, Jones M, Moore HD, Mason C, Andrews PW, Na J: Activation of pluripotency genes in human fibroblast cells by a novel mRNA based approach. PlOS ONE, 5:e14397.

8. Hanna J, Cheng AW, Saha K, Kim J, Lengner CJ, Soldner F, Cassady JP, Muffat J, Carey BW, Jaenisch R: Human embryonic stem cells with biological and 
epigenetic characteristics similar to those of mouse ESCs. Proc Natl Acad Sci USA 2010, 107:9222-9227.

9. Laurent LC, Ulitsky I, Slavin I, Tran H, Schork A, Morey R, Lynch C, Harness JV, Lee S, Barrero MJ, Ku S, Martynova M, Semechkin R, Galat V, Gottesfeld J, Izpisua Belmonte JC, Murry C, Keirstead HS, Park HS, Schmidt U, Laslett AL, Muller FJ, Nievergelt CM, Shamir R, Loring JF: Dynamic changes in the copy number of pluripotency and cell proliferation genes in human ESCs and iPSCs during reprogramming and time in culture. Cell Stem Cell 2011, 8:106-18.

10. Gore A, Li Z, Fung HL, Young J, Agarwal S, Antosiewicz-Bourget J, Canto I,
Giorgetti A, Israel M, Kiskinis E, Lee JH, Loh YH, Manos PD, Montserrat N, Panopoulos AD, Ruiz S, Wilbert M, Yu J, Kirkness EF, Izpisua-Belmonte JC, Ross: DJ, Thomson J, Eggan K, Daley GQ, Goldstein LSB, Zhang K: Somatic coding mutations in human induced pluripotent stem cells. Nature 2011, in press.

doi:10.1186/scrt46

Cite this article as: Li M, et al:. Cell fate conversion by mRNA. Stem Cell

Research \& Therapy 2011, 2:5. 